\title{
Universal Dephasing Rate due to Diluted Kondo Impurities
}

\author{
T. Micklitz, ${ }^{1}$ A. Altland, ${ }^{1}$ T. A. Costi, ${ }^{2}$ and A. Rosch ${ }^{1}$ \\ ${ }^{1}$ Institute for Theoretical Physics, University of Cologne, 50937 Cologne, Germany \\ ${ }^{2}$ Institut für Festkörperforschung, Forschungszentrum Jülich, 52425 Jülich, Germany
} (Received 12 October 2005; revised manuscript received 20 December 2005; published 7 June 2006)

\begin{abstract}
We calculate the dephasing rate due to magnetic impurities in a weakly disordered metal as measured in a weak-localization experiment. If the density $n_{\mathrm{S}}$ of magnetic impurities is sufficiently low, the dephasing rate $1 / \tau_{\varphi}$ is a universal function, $1 / \tau_{\varphi}=\left(n_{\mathrm{S}} / \nu\right) f\left(T / T_{\mathrm{K}}\right)$, where $T_{\mathrm{K}}$ is the Kondo temperature and $\nu$ is the density of states. We show that inelastic vertex corrections with a typical energy transfer $\Delta E$ are suppressed by powers of $1 /\left(\tau_{\varphi} \Delta E\right) \propto n_{\mathrm{S}}$. Therefore, the dephasing rate can be calculated from the inelastic cross section proportional to $\pi \nu \operatorname{Im} T-|\pi \nu T|^{2}$, where $T$ is the $T$ matrix which is evaluated numerically exactly using the numerical renormalization group.
\end{abstract}

PACS numbers: 72.15.Lh, 72.15.Qm, 72.15.Rn, 75.20.Hr

Dephasing, i.e., the loss of wave coherence, is a ubiquitous phenomenon in the quantum mechanics of complex systems. It is of relevance to any experiment where both interference and interactions play a role and is, therefore, of profound importance in all areas of nanoscopic and mesoscopic physics.

While the basic phenomenon of dephasing as such is of a rather general nature, the concrete definition of a dephasing rate and its experimental determination vary from context to context. In this Letter, we consider the dephasing rate as determined by weak-localization (WL) measurements in metals [1]. In weakly disordered metals, the interference of electronic wave functions on time-reversed paths leads to a characteristic reduction (or enhancement in the presence of spin-orbit interactions) of the conductivity. The magnitude of this effect is controlled by the dephasing time $\tau_{\varphi}$ - the typical time scale over which electrons get entangled with their environment (phonons, other electrons, or dynamical impurities), thereby losing the ability to interfere. Even small magnetic fields break time-reversal invariance, thus prohibiting the interference of time-reversed paths. Fitting the magnetoresistivity to WL theory is a means to determine the dephasing rate $1 / \tau_{\varphi}$ with high precision.

Surprisingly, most of these experiments [2] show a saturation of the dephasing rate at the lowest accessible temperatures $T$, while theoretically it is expected that in the limit $T \rightarrow 0$ all inelastic processes freeze out when the system approaches its (time-reversal invariant and unique) ground state. This has led to an intense discussion [2-5] as to whether quantum fluctuations can induce dephasing at $T=0$. While we believe that this latter scenario is theoretically excluded for electrons in a disordered metal, the presence of only a few parts per million of dynamical impurities - realized by atomic two-level systems [6] or by magnetic impurities [7-10] — may be an alternative cause of the saturation phenomenon. Indeed, it has been shown experimentally that magnetic impurities lead to an apparent saturation of $1 / \tau_{\varphi}$ at least in some $T$ range [9]. In contrast, some extremely pure $\mathrm{Au}$ and $\mathrm{Ag}$ samples with a negligible concentration of impurities [10] show no saturation and seem to follow the predictions of Altshuler, Aronov, and Khmelnitsky (AAK) [11] for the dephasing induced by Coulomb interactions.

The effect of dynamic magnetic impurities on $1 / \tau_{\varphi}$ was first considered by Ohkawa, Fukuyama, and Yosida [12] (for the static case, see Ref. [13]) using perturbation theory (generalized to renormalized perturbation theory in Refs. $[8,14])$, which limits the range of applicability to temperatures $T \gg T_{\mathrm{K}}$ larger than the Kondo temperature $T_{\mathrm{K}}$. For $T \ll T_{\mathrm{K}}$, a quadratic $T$ dependence $1 / \tau_{\varphi} \propto T^{2}$ has been predicted [14] based on Fermi liquid arguments. In this Letter, we argue that the impact of static and diluted dynamic impurities can largely be treated separately. This separation enables us to combine a perturbative approach to the static disorder with a (numerically) exact treatment of the Kondo interaction. In this way, the effect of a small concentration $n_{\mathrm{S}}$ of magnetic impurities on $1 / \tau_{\varphi}$ can be explored in the entire crossover range from $T \gg T_{\mathrm{K}}$ down to $T \ll T_{\mathrm{K}}$.

We consider the Hamiltonian $H=H_{0}+H_{\mathrm{S}}$, where

$$
H_{0}=\int d^{d} \mathbf{x} c_{\sigma}^{\dagger}(\mathbf{x})\left[\frac{\hat{\mathbf{p}}^{2}}{2 m}-\mu+V(\mathbf{x})\right] c_{\sigma}(\mathbf{x})
$$

describes electrons in a weak nonmagnetic disorder potential $V$, modeled for convenience by Gaussian white noise: $\left\langle V(\mathbf{x}) V\left(\mathbf{x}^{\prime}\right)\right\rangle_{V}=\frac{1}{2 \pi \nu \tau} \delta\left(\mathbf{x}-\mathbf{x}^{\prime}\right)$, where $\nu$ is the density of states per spin, $\tau=l / v_{\mathrm{F}}$ is the elastic scattering time, and $l$ is the elastic mean free path. (None of our results is affected by the precise choice of band structure or model of disorder.) The coupling of the electrons to a small concentration $n_{\mathrm{S}}$ of spin-1/2 impurities is described by the Kondo Hamiltonian

$$
H_{\mathrm{S}}=J \sum_{i} \hat{\mathbf{S}}_{i} c_{\sigma}^{\dagger}\left(\mathbf{x}_{i}\right) \sigma_{\sigma \sigma^{\prime}} c_{\sigma^{\prime}}\left(\mathbf{x}_{i}\right)
$$

To calculate physical quantities, we have (i) to average over $V(\mathbf{x})$ and (ii) the positions $\mathbf{x}_{i}$ of the spins, taking into account (iii) the exchange coupling $J$ to all orders. Two 
small parameters will help us in attacking this problem: The concentration of magnetic impurities $n_{\mathrm{S}}$ is tiny [more precisely, $\left.n_{\mathrm{S}} /\left(\nu T_{\mathrm{K}}\right) \ll 1\right]$ and also the ratio of electronic wavelength and elastic mean free path, $1 /\left(k_{\mathrm{F}} l\right)$, is small but finite.

We wish to explore the impact of dynamic impurity scattering on the WL corrections to the electric conductivity. Technically, this amounts to computing the impurity generated "self-energy" or "mass" of the Cooperon describing the coherent propagation of an electron and a time-reversed hole in the disordered environment. As justified in detail below, we neglect mixed interactiondisorder diagrams. The problem therefore reduces to the solution of the Bethe-Salpeter equation depicted diagrammatically in Fig. 1(a). To linear order in $n_{\mathrm{S}}$, the twoparticle irreducible vertex $\Gamma$ shown in Fig. 1(b) can be separated into three distinct contributions: self-energy diagrams [the first two terms in Fig. 1(b)], an "elastic" vertex correction with no energy transfer between upper and lower lines, and an "inelastic" vertex where interaction lines connect the two lines. We begin by focusing on the elastic contributions to the Cooperon self-energy as inelastic contributions give vanishingly small corrections for small $n_{\mathrm{S}}$; see below.

Since self-energy and elastic vertex contributions conserve the energy of single electron lines, the solution of the reduced Bethe-Salpeter equation amounts to a straightforward summation of a geometric series. Setting the centerof-mass frequency $\Omega$ (see Fig. 1) to 0 , the Cooperon obtains as $C_{\Omega=0}^{\mathbf{q}}\left(\epsilon, \epsilon^{\prime}\right)=\left(1 / 2 \pi \nu \tau^{2}\right) \delta\left(\epsilon-\epsilon^{\prime}\right) /\left[D \mathbf{q}^{2}+\right.$ $\left.1 / \tau_{\varphi}(\epsilon, T)\right]$, and the WL correction to the conductivity is

$$
\frac{\Delta \sigma_{\mathrm{WL}}}{\sigma_{\text {Drude }}}=2 \tau^{2} \int d \epsilon f^{\prime}(\epsilon) \int d^{d} \mathbf{q} \frac{1}{D \mathbf{q}^{2}+1 / \tau_{\varphi}(\epsilon)},
$$

with the $T$ and $\epsilon$ dependent dephasing rate

$$
\frac{1}{\tau_{\varphi}(\epsilon, T)}=\frac{2 n_{\mathrm{S}}}{\pi \nu}\left[\pi \nu \operatorname{Im}\left[T^{\mathrm{A}}(\epsilon)\right]-\left|\pi \nu T^{\mathrm{R}}(\epsilon)\right|^{2}\right] .
$$

The many-body $T$ matrix [defined by the Green function $\left.G_{\mathbf{x x}^{\prime}}(\epsilon)=G_{\mathbf{x x}^{\prime}}^{0}(\epsilon)+G_{\mathbf{x} \mathbf{0}}^{0}(\epsilon) T(\epsilon) G_{\mathbf{0} \mathbf{x}^{\prime}}^{0}(\epsilon)\right]$ describes the scat-

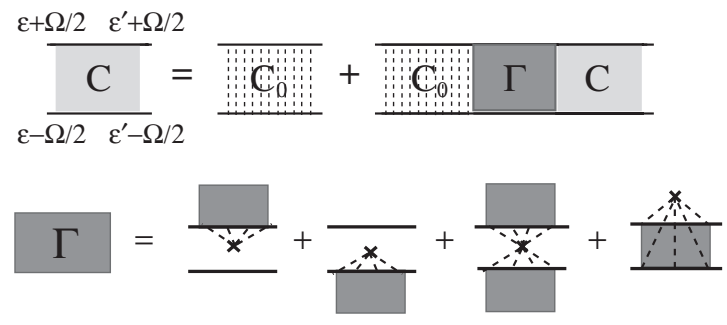

FIG. 1. Bethe-Salpeter equation for the Cooperon $C$ in the presence of (dilute) magnetic impurities to linear order in $n_{\mathrm{S}}$. $C_{0}$ is the bare Cooperon in the absence of interactions, and $\Gamma$ is the irreducible vertex obtained by adding self-energy, elastic, and inelastic vertex contributions. The crosses with attached dashed lines denote the averaging over impurity positions $\mathbf{x}_{i}$; the squares are the interactions to arbitrary order in $J$. tering of electrons from a single magnetic impurity. Equation (4) affords a simple interpretation: $\operatorname{Im}\left[T^{\mathrm{A}}(\epsilon)\right]$ is proportional to the total cross section, while $\left|T^{\mathrm{R}}(\epsilon)\right|^{2}$ describes the elastic cross section. Their difference is - by definition-proportional to the inelastic cross section recently introduced by Zaránd et al. [15]. Note that the optical theorem guarantees the vanishing of the inelastic cross section for static impurities. Together with the numerical evaluation of $1 / \tau_{\varphi}$ and the analytic estimates of the leading corrections, given below, Eq. (4) is the main result of this Letter.

To determine the range of applicability of Eq. (4), three classes of corrections have to be considered: contributions from the inelastic vertex, mixed interaction-disorder diagrams, and higher-order corrections in $n_{\mathrm{S}}$. We begin by considering the lowest order expansion of the WL correction in the inelastic vertex [cf. Fig. 2(b)]

$$
\Delta \sigma_{1}=\int d \epsilon \int d \Omega \tilde{f}(\epsilon, \Omega) \Gamma_{\mathrm{in}}(\epsilon, \Omega) I_{d}(\epsilon, \Omega) .
$$

Here $\tilde{f}$ denotes some thermal function restricting $\epsilon$ and $\Omega$ to values smaller than $T, \Gamma_{\text {in }}$ is the inelastic vertex, and $I_{d}(\epsilon, \Omega) \sim\left[\left(1 / \tau_{\varphi}\right)^{2}+\Omega^{2}\right]^{d-4 / 4}$ results from momentum integration over two Cooperons in $d$ dimensions. For sufficiently small $n_{\mathrm{S}} \ll \nu T_{\mathrm{K}}$, the typical energy $\Delta E \sim \Omega \sim T$ [16] exchanged at the inelastic vertex greatly exceeds the dephasing rate (see below) $1 / \tau_{\varphi} \propto n_{\mathrm{S}}$. Evaluating the integral (5) for $\Delta E \gg 1 / \tau_{\varphi}$, we obtain

$$
\frac{\Delta \sigma_{1}}{\Delta \sigma_{\mathrm{WL}}} \sim\left(\frac{1}{\Delta E \tau_{\varphi}}\right)^{(4-d) / 2} \lesssim\left(\frac{n_{\mathrm{S}}}{\nu T_{\mathrm{K}}}\right)^{(4-d) / 2} \ll 1 .
$$

It is straightforward to verify that this estimate pertains to higher orders in the expansion in the inelastic vertex.

The irrelevancy of the inelastic vertex for processes with typical energy transfer larger than $1 / \tau_{\varphi}$ has been observed before by several authors (e.g., $[11,14,17])$ : Semiclassically, vertex corrections describe the interference of two (time-reversed) electrons undergoing an interaction process [see Fig. 2(a)]. Because of the transferral of a certain energy $\Delta E$, the two electrons subsequently accumulate a phase difference $e^{i \Delta E t}$, where $t \sim \tau_{\varphi}$. Therefore, (a)

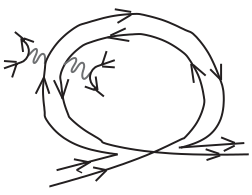

(c)

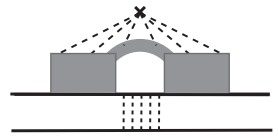

(b)

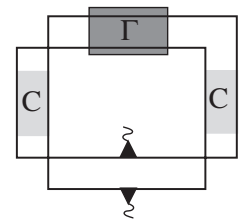

(d)

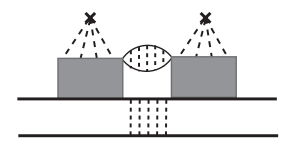

FIG. 2. (a) Interference of interacting electrons. (b) Corrections due to the inelastic vertex to lowest order. (c) WL correction to $\tau_{\varphi}^{-1}$ linear in $n_{\mathrm{S}}$. (d) AAK type corrections arising to order $n_{\mathrm{S}}^{2}$. 
vertex corrections with $\Delta E>1 / \tau_{\varphi}$ (characteristic for our problem) do not effectively contribute to WL. (In contrast, Coulomb interactions in $d \leq 2$ are dominated by lowenergy transfers and vertex corrections are required to regularize infrared singularities [11].)

We next turn to the discussion of the second family of corrections potentially altering our above results, correlated disorder-interaction processes as shown in Fig. 2(c). A preliminary indication as to the relevance of such contributions may be obtained by estimating the sample-tosample fluctuations [18] of the Kondo temperature $T_{\mathrm{K}}$,

$$
\left(\frac{\delta T_{\mathrm{K}}}{T_{\mathrm{K}}}\right)^{2}=\frac{1}{\nu^{2}} \int_{T_{\mathrm{K}}}^{E_{\mathrm{F}}} d \omega \int_{T_{\mathrm{K}}}^{E_{\mathrm{F}}} d \omega^{\prime} \frac{\left\langle\delta \nu(\omega) \delta \nu\left(\omega^{\prime}\right)\right\rangle_{V}}{\omega \omega^{\prime}} .
$$

Substituting the results for the fluctuations of the local density of states $\delta \nu$ in weakly disordered $d$-dimensional metals [1], we obtain

$$
\left(\frac{\delta T_{\mathrm{K}}}{T_{\mathrm{K}}}\right)^{2} \sim \begin{cases}\frac{1}{\left(k_{\mathrm{F}} L_{\perp}\right)^{2}} \frac{1}{\sqrt{\tau T_{\mathrm{K}}}} & \text { in (quasi) } d=1, \\ \frac{1}{k_{\mathrm{F}} l} \frac{1}{(J \nu)^{3}} & \text { in } d=2, \\ \frac{1}{\left(k_{\mathrm{F}} l\right)^{2}} \frac{1}{(J \nu)^{2}} & \text { in } d=3,\end{cases}
$$

where $L_{\perp}^{2}$ is the cross section of a quasi- $1 d$ wire. We assume, henceforth, that $k_{\mathrm{F}} l$ is sufficiently large, so that $\delta T_{\mathrm{K}} \ll T_{\mathrm{K}}$. While this condition seems restrictive in quasi- $1 d$, it turns out to be always met in the WL regime $\Delta \sigma^{\mathrm{WL}} \ll \sigma^{\text {Drude }}$, realized in experiments (and assumed here).

More formally, the role of correlations in disorder or interactions may be explored in terms of the diagrams shown in Fig. 2(c). On the face of it, these diagrams are smaller by factors of $1 /\left(k_{F} l\right)$ than the leading contributions considered above (as quantum interference maintained across the impurity limits the momentum exchanged to values $\lesssim l^{-1}$ much smaller than $k_{F}$ ). However, for very low $T$ the enhanced infrared singularity caused by the presence of extra diffusion modes may overcompensate this phase space suppression factor. Using that for $T \ll$ $T_{\mathrm{K}}$ the bare interaction may be described by Fermi liquid theory [19], we find that only in one dimension do the additional diagrams $\left(\propto T^{(d+2) / 2}+T^{2}\right)$ lead to contributions of anomalously strong singularity. Specifically, we obtain a correction to the dephasing rate $1 / \tau_{\varphi, c} \sim n_{\mathrm{S}} T^{3 / 2} /$ $\left[\nu T_{\mathrm{K}}^{2} \sqrt{\tau}\left(k_{F} L_{\perp}\right)^{2}\right]$. Therefore, for

$$
T \lesssim \frac{1}{\left(k_{\mathrm{F}} L_{\perp}\right)^{4}} \frac{1}{\tau T_{\mathrm{K}}} T_{\mathrm{K}} \ll T_{\mathrm{K}},
$$

the separation disorder or interactions used above become invalid in $d=1$.

At very low $T$, yet another type of correction begins to play a role: The diluted Kondo impurities become indistinguishable from a conventional disordered Fermi liquid with short-range momentum-conserving interactions [20] and the dephasing rate is determined by AAK $[11,20]$ type processes which, in our context, are encapsulated in the third family of diagrams shown in Fig. 2(d). Contributing only at order $n_{\mathrm{S}}^{2}$, they generate corrections scaling as $T^{2 / 3}$, $T$, and $T^{3 / 2}$ in $d=1,2,3$, respectively. Evaluating the prefactors (again in Fermi liquid theory), we find that these contributions become sizable for

$$
T \lesssim \begin{cases}\frac{1}{\left(k_{\mathrm{F}} l\right)^{4}}\left(\frac{n_{\mathrm{S}}}{\nu T_{\mathrm{K}}}\right)^{2} \tau T_{\mathrm{K}}^{2} & d=3, \\ \frac{1}{k_{\mathrm{F}} l} \frac{n_{\mathrm{S}}}{\nu T_{\mathrm{K}}} T_{\mathrm{K}} & d=2, \\ \frac{1}{k_{\mathrm{F}} L_{\perp}} \frac{1}{\left(\tau T_{\mathrm{K}}\right)^{1 / 4}}\left(\frac{n_{\mathrm{S}}}{\nu T_{\mathrm{K}}}\right)^{1 / 4} T_{\mathrm{K}} & d=1 .\end{cases}
$$

In all cases, the crossover scale is well below $T_{\mathrm{K}}$ and may arguably be neglected in all relevant experiments. Further corrections to $1 / \tau_{\varphi}$ of order $n_{\mathrm{S}}^{2}$ and higher arise from clusters of two and more magnetic impurities which are sufficiently close that the interimpurity coupling dominates over the Kondo effect [21].

Fitting to $\Delta \sigma=2 \tau^{2} \sigma_{\text {Drude }} \int d^{d} \mathbf{q}\left(D \mathbf{q}^{2}+\tau_{\varphi}^{-1}(T)\right)^{-1}$, we next relate our results to the $T$-dependent dephasing rates $\tau_{\varphi}^{-1}(T)$ extracted from experiments. Comparison with the energy-resolved representation (3) results in

$$
\frac{1}{\tau_{\varphi}(T)}= \begin{cases}{\left[-\int d \epsilon f^{\prime}(\epsilon) \tau_{\varphi}(\epsilon, T)^{(2-d) / 2}\right]^{2 /(d-2)}} & d=1,3, \\ \exp \left[\int d \epsilon f^{\prime}(\epsilon) \ln \tau_{\varphi}(\epsilon, T)\right] & d=2, \\ -\int d \epsilon f^{\prime}(\epsilon) / \tau_{\varphi}(\epsilon, T) & \omega_{B} \tau_{\varphi} \gg 1,\end{cases}
$$

where the last line applies to the case where a strong magnetic field is present and the Cooperon can be expanded in $1 /\left(\omega_{B} \tau_{\varphi}\right)$. ( $\omega_{B}$ is the "cyclotron" frequency of the Cooperon.)

Just two parameters enter the expression for $1 / \tau_{\varphi}$ : the Kondo temperature $T_{\mathrm{K}}$ and the dimensionless density of magnetic impurities $n_{\mathrm{S}} /\left(\nu T_{\mathrm{K}}\right) \ll 1$. The $T$ matrix in Eq. (4) can be evaluated for arbitrary values of $T / T_{\mathrm{K}}$ and $\epsilon / T_{\mathrm{K}}$ us-

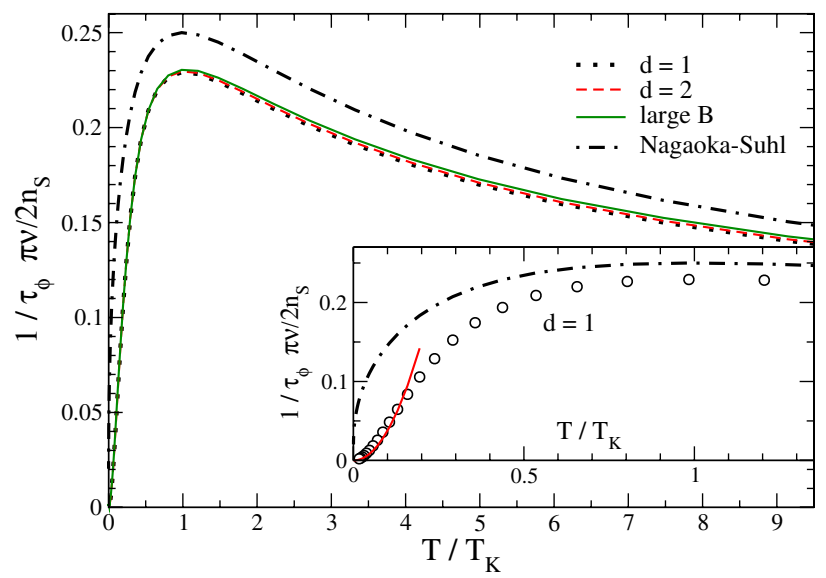

FIG. 3 (color online). Universal dephasing rate calculated via NRG. Accidentally, there is almost no dependence on the dimension $d$. The dotted-dashed line shows the result of a Nagaoka-Suhl resummation [26] $1 / \tau_{\varphi}=\left(n_{\mathrm{S}} / 2 \pi \nu\right)\left[\left(\pi^{2} 3 / 4\right) /\right.$ $\left.\left(\pi^{2} 3 / 4+\ln ^{2} T / T_{\mathrm{K}}\right)\right]$, using our definition of $T_{\mathrm{K}}$ [25]. While the Fermi liquid behavior is recovered for $T \lesssim 0.1 T_{\mathrm{K}}$, the calculated prefactor of the $T^{2}$ behavior (solid line) is not exactly reproduced due to numerical problems [23]. 
ing the numerical renormalization group (NRG) [22] generalized to the calculation of dynamical quantities in Refs. [23,24]. The result is shown in Fig. 3, where we define [25] and determine the Kondo temperature $T_{\mathrm{K}}=T_{\mathrm{K}}^{(0)}$ from the $T=0$ susceptibility $\chi=\left(g \mu_{B}\right)^{2} /\left(4 T_{\mathrm{K}}^{(0)}\right)$. For $T \gg T_{\mathrm{K}}$, we obtain (correcting by factors of 2 in Ref. [8] and 16 in Ref. [14]) $1 / \tau_{\varphi} \approx 3 \pi n_{\mathrm{S}} /\left(8 \nu \ln ^{2}\left[T / T_{\mathrm{K}}\right]\right)$, giving rise to a very weak $T$ dependence for $T \geqslant T_{\mathrm{K}}$. For $T \lesssim$ $0.3 T_{\mathrm{K}}$, one observes a crossover regime where $1 / \tau_{\varphi}$ varies almost linear in $T$, while at lowest $T$ one obtains the expected $T^{2}$ behavior. This low-temperature regime can also be calculated analytically using Fermi liquid theory [19],

$$
\frac{1}{\tau_{\varphi}(T)} \approx c_{\mathrm{FL}} \frac{2 n_{\mathrm{S}}}{\pi \nu}\left(\frac{T}{T_{\mathrm{K}}}\right)^{2} \frac{\pi^{4}}{24},
$$

with $c_{\mathrm{FL}} \approx 0.927,0.946,0.969$ in $d=1,2,3$ and $c_{\mathrm{FL}}=1$ for $\omega_{B} \tau_{\varphi} \gg 1$ (correcting prefactors in Ref. [14]). Accidentally, the dependence of both the analytical and numerical results on $\omega_{B} \tau_{\varphi}$ (and on $d$ ) is very weak, implying that the usual fits of the magnetoresistivity can be used to determine $1 / \tau_{\varphi}$.

In the comparison to concrete experiments, one needs to account for the interplay of dephasing due to magnetic impurities and due to Coulomb interactions [11]. Since the latter are controlled by infrared divergences in $d \leq 2$, the respective rates do not add. Instead, one needs to solve, e.g., in quasi-one-dimensional systems, the equation

$$
\frac{1}{\tau_{\varphi}}=\kappa T \sqrt{\tau_{\varphi}}+\frac{1}{\tau_{\varphi, \mathrm{S}}} \approx\left\{\begin{array}{l}
(\kappa T)^{2 / 3}+2 /\left(3 \tau_{\varphi, \mathrm{S}}\right) \\
1 / \tau_{\varphi, \mathrm{S}}+\kappa T \sqrt{\tau_{\varphi, \mathrm{S}}}
\end{array}\right.
$$

where the first term describes the self-consistently calculated effects of Coulomb interactions, while $1 / \tau_{\varphi, \mathrm{S}}$ is the dephasing rate due to the magnetic impurities. The first (second) line holds when the Coulomb dephasing (Kondo dephasing) dominates.

In conclusion, we have shown that the dephasing rate due to diluted magnetic impurities can be calculated directly from the inelastic cross section (4) introduced in Ref. [15]. This result is valid for all types of diluted dynamical impurities as long as typical energy transfers remain larger than $1 / \tau_{\varphi}$. In the case of Kondo impurities, the dephasing rate depends on just two parameters, the Kondo temperature $T_{\mathrm{K}}$ and the dimensionless density of magnetic impurities $n_{\mathrm{S}} /\left(\nu T_{\mathrm{K}}\right) \ll 1$. A measurement of $\tau_{\varphi}$ for spin-1/2 impurities along with an independent experimental determination of $T_{\mathrm{K}}$ and $n_{\mathrm{S}}$ would put our theory to a parameter-free test [up to fitting the Coulomb background, Eq. (12)]. An open question is how spin-orbit interactions and disorder influence the Kondo effect in systems with larger spins, e.g., Fe in Au [9].

We acknowledge discussions with $\mathrm{Ch}$. Bäuerle, L. Glazman, J.v. Delft, S. Mirlin, B. Spivak, and P. Wölfle and financial support of the DFG by SFB 608 and TR 12.
[1] B.L. Altshuler and A.G. Aronov, in Electron-Electron Interaction in Disordered Systems, edited by A.L. Efros and M. Pollak (North-Holland, Amsterdam, 1985).

[2] P. Mohanty, E. M. Q. Jariwala, and R. A. Webb, Phys. Rev. Lett. 78, 3366 (1997); P. Mohanty and R. A. Webb, Phys. Rev. B 55, R13 452 (1997).

[3] D. S. Golubev and A. D. Zaikin, Phys. Rev. Lett. 81, 1074 (1998).

[4] I.L. Aleiner, B.L. Altshuler, and M.E. Gershenson, Waves Random Media 9, 201 (1999).

[5] J. v. Delft, J. Phys. Soc. Jpn. 72A, 24 (2003).

[6] A. Zawadowski, J.v. Delft, and D.C. Ralph, Phys. Rev. Lett. 83, 2632 (1999).

[7] P. Mohanty and R. Webb, Phys. Rev. Lett. 84, 4481 (2000).

[8] C. Van Haesendonck, J. Vranken, and Y. Bruynseraede, Phys. Rev. Lett. 58, 1968 (1987).

[9] F. Schopfer et al., Phys. Rev. Lett. 90, 056801 (2003).

[10] F. Pierre et al., Phys. Rev. B 68, 085413 (2003).

[11] B.L. Altshuler, A. G. Aronov, and D.E. Khmelnitsky, J. Phys. C 15, 7367 (1982).

[12] F. J. Ohkawa, H. Fukuyama, and K. Yosida, J. Phys. Soc. Jpn. 52, 1701 (1983); F. J. Ohkawa, Prog. Theor. Phys. Suppl. 84, 166 (1985).

[13] S. Hikami, A. I. Larkin, and Y. Nagaoka, Prog. Theor. Phys. 63, 707 (1980).

[14] M. G. Vavilov and L. I. Glazman, Phys. Rev. B 67, 115310 (2003); M. G. Vavilov, L. I. Glazman, A. I. Larkin, Phys. Rev. B 68, 075119 (2003).

[15] G. Zaránd, L. Borda, J. v. Delft, and N. Andrei, Phys. Rev. Lett. 93, 107204 (2004).

[16] More precisely, $\Delta E \approx T$ in the Fermi liquid regime $T \ll$ $T_{\mathrm{K}}$, while for $T \gg T_{\mathrm{K}}, \Delta E \approx T / \ln ^{2}\left[T / T_{\mathrm{K}}\right]$ is given by the lifetime of the spin. Comparing this with $1 / \tau_{\varphi} \sim n_{\mathrm{S}} / \nu$ at $T \sim T_{\mathrm{K}}$, we obtain the criterion stated in the text.

[17] J. S. Meyer, V.I. Fal'ko, and B.L. Altshuler, in Strongly Correlated Fermions and Bosons in Low-Dimensional Disordered Systems, edited by I. V. Lerner et al., Nato Science Series II Vol. 72 (Kluwer Academic, Dordrecht, 2002).

[18] S. Kettemann and E. R. Mucciolo, JETP Lett. 83, 240 (2006).

[19] A.C. Hewson, The Kondo Problem to Heavy Fermions (Cambridge University Press, Cambridge, England, 1993).

[20] B. N. Narozhny, Gábor Zala, and I.L. Aleiner, Phys. Rev. B 65, 180202(R) (2002).

[21] G. Frossati et al., Physica (Amsterdam) 84B+C, 33 (1976).

[22] K. G. Wilson, Rev. Mod. Phys. 47, 773 (1975).

[23] T. A. Costi, A. C. Hewson, and V. Zlatic, J. Phys. Condens. Matter 6, 2519 (1994).

[24] W. Hofstetter, Phys. Rev. Lett. 85, 1508 (2000).

[25] In the literature [19], a number of other definitions of $T_{\mathrm{K}}$ are used. The perturbative $T_{\mathrm{K}}$ is given by $0.4107 T_{\mathrm{K}}^{(0)}[19]$; the half width at half maximum of the spectral function at $T=0$ is $\approx 2.05 T_{\mathrm{K}}^{(0)}$. At $T \approx 0.94 T_{\mathrm{K}}^{(0)}\left(T \approx 0.9 T_{\mathrm{K}}^{(0)}\right)$, the resistivity of diluted Kondo impurities in a dirty (clean) metal and the conductance through a quantum dot have half of their maximal value.

[26] Correcting a factor of 2 in Ref. [8]. 